A\&A 369, 291-304 (2001)

DOI: 10.1051/0004-6361:20010142

(c) ESO 2001

\title{
Active region transient brightenings
}

\section{A simultaneous view by SXT, EIT and TRACE}

\author{
D. Berghmans ${ }^{1}$, D. McKenzie ${ }^{2}$, and F. Clette ${ }^{1}$ \\ 1 Royal Observatory of Belgium, Ringlaan 3, 1180 Brussel, Belgium \\ 2 Department of Physics, PO Box 173840, Montana State University, Bozeman, MT 59717-3840, USA
}

Received 30 October 2000 / Accepted 29 January 2001

\begin{abstract}
This paper reports on a qualitative study on the weakest flarelike brightenings in active region that can be observed with current coronal imagers. Specificallly, we investigate the correspondence of the "active region transient brightenings" (ARTB) first observed almost a decade ago with SXT with similar brightenings in the EUV that are now observed by EIT and TRACE. For this goal, exceptionally high cadence image sequences were acquired of a small but rapidly evolving active region (NOAA 8218), simultaneously by SXT, EIT, and TRACE. Within the timeframe of this coordinated campaign, we detected 41 soft X-ray brightenings and 373 EUV brightenings. We find that the strongest brightenings observed by EIT are indeed the EUV counterparts of the ARTBs seen by SXT. Weaker brightenings seen by EIT often do not have an X-ray counterpart. Among the brightenings detected with SXT we discover a new subpopulation, consisting of events that brighten in soft X-rays only, at a footpoint of a pre-existing SXT loop shortly after an ARTB occurred at the other footpoint. The propagation speed of the perturbation suggests an interpretation in terms of slow mode MHD waves.
\end{abstract}

Key words. Sun: activity - Sun: corona - Sun: flares - Sun: UV radiation - Sun: X-rays

\section{Introduction}

Flares are the most violent manifestation of solar activity, releasing in only a few minutes huge amounts of energy over the entire electromagnetic spectrum. It has been realised however that despite the enormous release of energy in big flares, they are so rare that their time-averaged power contribution is too small to account completely for heating of the solar corona. The most energetic flares, however, are just the tip of the iceberg and progressively more flarelike events are observed for smaller energy releases. Collectively, these weakest events might contribute the bulk of the required power to keep the corona at its high temperature.

Using the Hard X-Ray Imaging Spectrometer (HXIS) onboard the Solar Maximum Mission (SMM), Schadee et al. (1983) were the first to describe observations of short-lived X-ray brightenings with hard X-ray fluxes $10^{-3}$ smaller than regular flares. Shortly later, Lin et al. (1984) made similar observations from balloon-borne detectors. On the basis of soft X-ray image sequences of the Soft X-ray telescope (SXT, on board Yohkoh), Shimizu and co-workers were able to clearly identify a set of events

Send offprint requests to: D. Berghmans,

e-mail: David.Berghmans@ksb-orb.oma. be that they called "Active region transient brightenings" (ARTBs) (e.g. Shimizu et al. 1992, 1994; Shimizu \& Tsuneta 1997). They take the form of sudden brightenings of magnetic loops and last from a few minutes to tens of minutes. They have a thermal energy content in the range $10^{25}$ to $10^{29} \mathrm{ergs}$, and their frequency of ocurrence (1-40 events per hour per active region) has a strong correlation to the total soft X-ray flux of the active region (Shimizu et al. 1992).

To demonstrate that ARTBs show, just as do "regular" flares, evidence for the acceleration of nonthermal electrons, the soft X-ray observations of ARTB have been combined with simultaneous hard X-ray and radio observations (Gopalswamy et al. 1994, 1997; White et al. 1995; Nindos et al. 1999). The hard X-ray emission of the ARTBs is most noticeable during the rise phase. Just as for regular flares, this hard X-ray emission is most likely explained by precipitating electrons thermalizing in the chromosphere (Nitta 1997). The non-thermal radio emission in the rise phase is presumably due to gyrosynchotron emission of relativistic electrons (Gary et al. 1997). In ARTBs, the delay of soft X-rays relative to centimeter radio emission and hard X-ray emission is between 1.5 and $6.4 \mathrm{~min}$, similar to the well known delay in regular impulsive flares (Neupert 1968). Similar results were found 
with the aid of VLA observations for small scale network flares (Krucker et al. 1997). This Neupert effect is generally interpreted as flare energy release in the corona accompanied by accelerating particles, which propagate to the dense layer below where they deposit some of the flare energy. The chromospheric plasma is heated to coronal temperatures, expands and fills up loops that reach into the corona (referred to as "evaporation"). Combining all these results, one arrives to the unavoidably conclusion that ARTBs are indeed most likely scaled down flares, the biggest among them corresponding to the GOES class B flares.

When the rate of occurrence of ARTBs is examined as a function of their energy (Shimizu et al. 1994, 1995), the distribution is in the shape of a powerlaw with index 1.5-1.6. This is similar to the powerlaw with a slope of 1.53 found for the distribution of total energy in solar flares Crosby et al. (1993). It was shown (Hudson 1991) that in order to explain the heating of the corona by microor nanoflares (flarelike events with an energy output respectively $10^{-6}$ and $10^{-9}$ smaller than regular flares), the number distribution of small energy release events must be a power law with index steeper than 2. Extensive statistics of the smallest flarelike phenomena are therefore needed. The wavelength at which such events are best observed depends on the magnitude of the event. Porter et al. (1995, and Ref. therein) found that the X-ray emission from microflares scales as a power 2.32 of the corresponding EUV emission (CIV). Nitta (1997) found that the hard X-ray output scales as the power 1.51 of the soft X-ray output of ARTB. Since these values for the power are larger than 1 , it means that as we go to weaker events, their peak emission happens at longer wavelengths. Combining results from different authors, Aschwanden (1999) showed that the typical lengthscale of brightenings in the solar corona scales linearly with the temperature and that the total observed emission measure even scales as the seventh power of the temperature.

These combined facts indicate that a thorough investigation of the smallest events in the solar flare population should include a comparison of their soft X-ray emission with their EUV emission. From earlier space missions like SMM, Skylab or HRTS it was already known that EUV "bursts" or impulsive variations occur nearly continuously in active regions. Withbroe et al. (1985) and Habbal et al. (1985) report brightenings in EUV emission lines lasting between 5 and $15 \mathrm{~min}$ and spatial scales between $5^{\prime \prime}$ and $15^{\prime \prime}$. Since the launch of SOHO, the Extreme Ultraviolet Imaging Telescope (EIT) and the Coronal Diagnostics Spectrometer (CDS) frequently observe shortlived brightenings in active regions in the EUV. These were noted as very rapid time variability in active region loops (e.g. Kjeldseth-Moe \& Brekke 1998). Berghmans \& Clette (1999) reported on transient EUV brightenings with very similar characteristics as the ARTBs seen in soft X-rays. Currently, the most advanced studies are made with the EUV imager onboard the Transition Region and Coronal Explorer (TRACE). The high spatial resolution
(0.5 arcsec per pixel) allows for the detection of the smallest coronal brightenings yet observed (Nightingale et al. 1999; Aschwanden et al. 2000). Although a lot of authors thus describe active region transient brightenings and refer to the above quoted work by Shimizu and colleagues, no detailed comparison has been done yet - to the best of our knowledge - that compares event per event, ARTBs with the brightenings observed in active regions by present day EUV imagers such as EIT or TRACE.

The simultaneous operation of three superb instruments like SXT, EIT, and TRACE therefore provides unprecedented opportunities. In this respect, SOHO JOP 80 (Clette et al. 1998; Berghmans \& Clette 1999) was designed to exploit the simultaneous observations of these three imagers in complementary passbands. In order to detect as many as possible of the weak, short-lived brightenings, an effort was made to operate the imagers at their highest possible cadence. The EIT dataset (195 A bandpass) was already examined separately (Berghmans \& Clette 1999) and revealed hundreds of active region EUV brightenings. In this paper, we want to further explore the small-scale end of the ARTB population. The questions we want to answer are

- How are the soft X-ray ARTBs related to EUV brightenings? Do ARTBs exist without EUV counterparts? Do EUV brightenings exist without soft $X$-ray counterparts?

- What are the smallest ARTBs detectable with SXT? Can we find yet smaller events in the EUV?

Therefore we first show, in Sect. 3, that the acquired SXT data contain brightenings which, based on their physical characteristics, appear to be the same as Shimizu's ARTBs. When detecting brightenings, we use an automated detection tool which is more sophisticated than what was previously used. In Sect. 4, we study the EUV counterparts of these soft X-ray ARTBs. This is first done from a global perspective, by comparing the set of ARTBs with the set of EUV brightenings observed in the active region. Secondly, we examine in detail the EUV signature of individual ARTBs. Finally, in Sect. 5 we summarize and discuss the main results of this paper.

\section{The data}

On May 13, 1998, active region NOAA 8218 (see Fig. 1) was observed during the multi-instrument campaign "SOHO JOP 80" (see Berghmans \& Clette 1999). In the 24 hours preceeding the JOP 80 campaign, region 8218 , originally in a $\beta$ configuration, grew more complex due to the emergence of new bipolar areas within the leading portion of the group. This resulted in C-class flaring on May 23, while during the JOP80 campaign the activity was limited to B-class flaring. In this paper we use a subset of the JOP80 data set, viz. the images collected by the SXT, EIT, and TRACE imagers. Additional context information is gained from MDI magnetograms, as well as GOES-8 data. 

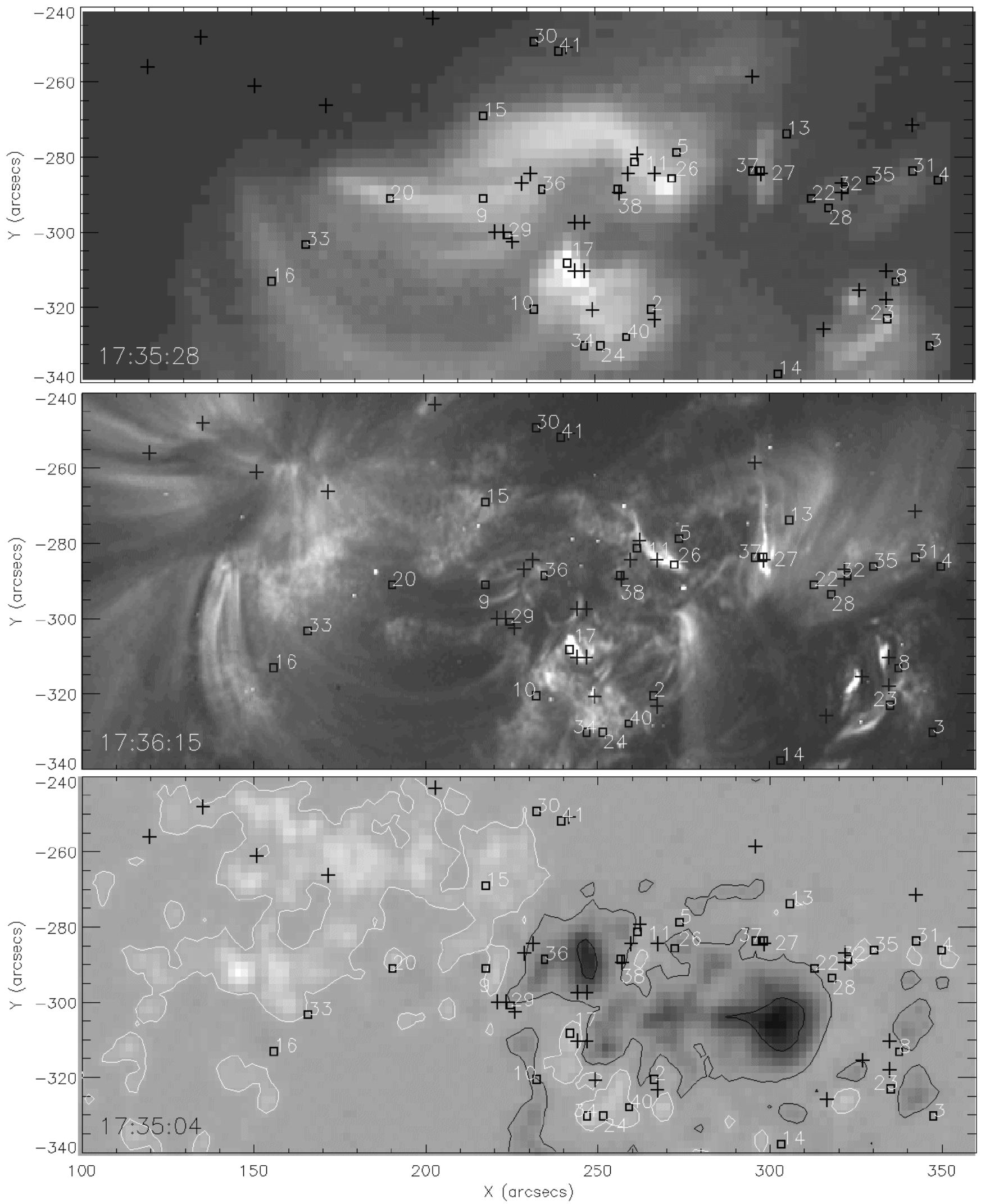

Fig. 1. Nearly simultaneous images of AR 8218 by SXT (top) and TRACE (middle), and a MDI magnetogram (below). The contours on the MDI magnetogram encircle regions below -100 Gauss (dark) and above +100 Gauss (bright) respectively. The numbered squares refer to the SXT ARTBs listed in Table 1. The crosses correspond to the location of EUV brightenings detected in the simultaneous EIT data. At the time of these images, ARTB 17, 26, 27 and 23 are approaching their peak intensity 
The SXT (Tsuneta et al. 1991) onboard the Yohkoh satellite (Ogawara et al. 1991) makes use of a grazing incidence mirror and 2.45 arcsec pixels. The field of view was $5.2 \times 5.2$ arcmin centered around NOAA 8218. Between $17 \mathrm{~h} 21$ and $17 \mathrm{~h} 57 \mathrm{UT}$, we collected 229 images through the $\mathrm{Al} / \mathrm{Mg} / \mathrm{Mn}$ filter, interleaved with 23 images through the Al 1265 A filter (a.k.a. "AlMg" and "Al.1" filters, respectively). It is possible to infer a mean electron temperature and emission measure from ratios of images taken through the various filters. The filter pair chosen is most useful for temperatures between about 1 and 5 MK. Because the AlMg filter images have far less stray light, the observations were weighted in favor of that filter: AlMg:Al.1 = 10:1. This means that while the AlMg images come at a cadence of one per $8 \mathrm{~s}$, the Al.1 images (and thus the temperatures estimates) are at a cadence of approximately $98 \mathrm{~s}$.

EIT (Delaboudinière et al.1995) imaged through its FeXII (195 $\AA$ ) bandpass (peak formation temperature: $1.610^{6} \mathrm{~K}$ ) from $17 \mathrm{~h} 32$ up to $18 \mathrm{~h} 29$. EIT was run in "shutterless mode" to increase the cadence up to $15 \mathrm{~s}$ (230 images). In "shutterless mode", the mechanical shutter is not operated and is kept in the open position for the duration of the whole observing sequence. The "BLOCK EAST" position of the filter wheel is used, which effectively limits the exposure to the western $2 / 3$ of the CCD. The masked eastern $1 / 3$ of the CCD is used as a storage area. This setup allows one to use EIT in a frame-transfer mode, thereby increasing the cadence. The spatial resolution of EIT is determined by its pixel size of 2.59 arcsec. The field of view was 5.5 arcmin by 4.1 arcmin.

TRACE (Tarbell et al. 1994; Handy et al. 1999) observed in the complementary Fe IX-X (171 $\AA$ ) bandpass (peak formation temperature: $1.010^{6} \mathrm{~K}$ ) from $17 \mathrm{~h} 00$ to $19 \mathrm{~h} 00$ with a nominal cadence of $25 \mathrm{~s}$, counting in total 287 images with a spatial resolution of 0.5 arcsec per pixel. The TRACE images were preprocessed using the standard procedures described in the SolarSoft TRACE analysis guide (Bentley 2000). A significant number of the TRACE images was heavily corrupted by particle hits. As a consequence of this, we did not attempt to detect brightenings directly in the TRACE dataset. However, we do take benefit of the higher spatial resolution of TRACE, when we visually check the EUV signature of each ARTB detected in the SXT sequence.

\section{Identification of ARTB}

Besides straight visual inspection, Shimizu (1995) collected ARTB from SXT data with a "macropixel method": an average lightcurve is extracted for each group of 16 by 16 pixels (a macropixel). If the lightcurve of a macropixel increases by more than 3 sigma (Poisson statistics) in one timestep, an "event" is identified. In our view, this method has two drawbacks. First, this method averages over a number of pixels which smooths out the smallest and/or weakest events. Secondly, if an hypothetical lightcurve is constant at all times except for 1 dip, the increase after the dip will result in an erroneous detection.

Therefore, we employ a different detection scheme that detects localised transient brightenings. The crux of this method is the calculation of a pixelwise "background" emission level to which emission enhancements or brightenings are compared. In an iterative procedure, the background level is re-estimated, as progressively more brightenings are identified, in order to remove any positive bias due to the transients (see Berghmans et al. 1998; Berghmans \& Clette 1999 for all technical details).

In the SXT data sequence, we looked for events with a peak intensity exceeding the calculated background level at the $\Sigma_{\mathrm{P}}=5 \sigma_{\mathrm{o}}$ significance level, where $\sigma_{\mathrm{o}}$ is the pixelwise standard deviation measuring the variability around the "background" emission level. Neighbouring pixels are assumed to be part of the same event if their light curve exceeds the $\Sigma_{\mathrm{E}}=3 \sigma_{\mathrm{o}}$ significance level simultaneously. The duration of the event is traced backwards and forwards in time until the light curves involved go below the $\Sigma_{\mathrm{D}}=2 \sigma_{\mathrm{o}}$ significance level. In order to eliminate unreliable, marginal events, we impose a final condition that only events at least 5 SXT pixels in spatial extent are retained. These criteria resulted in the 41 soft X-ray brightening events listed in Table 1 . Relaxation of the size criterion yields 12 more events which are probably almost all noise fluctuations (see Fig. 11 for a counterexample).

All of these 41 events have been visually inspected. Three events were identified as instrumental effects at the occasional mismatch of two partial frames that together form our SXT images. Based on the 38 remaining events observed in 36 min of SXT data, we arrive at an occurrence rate of about 1 event per minute for active region NOAA 8218. Shimizu (1992) reported a frequency of ocurrence of ARTB between 1-40 events per hour per active region, with the higher occurrence rates corresponding to the brightest active regions (integrated soft X-ray flux over the whole active region). Taking into account that the total soft X-ray flux (through the Al $1265 \AA$ filter) of NOAA 8218 was about $2.710^{6} \mathrm{DN} / \mathrm{s}$, the trend reported by Shimizu (1992) suggests that we should find between 2 and 10 events per hour. Our occurrence rate of about 1 event per minute is significantly above this expectation. Possible reasons for this discrepancy are (1) the exceptionally high cadence of the present dataset, (2) the more performant brightening detection method that we used or (3) an intrinsically higher variability of this particular active region.

In what follows, we will exclude five more events because these events were already decaying when the SXT image sequence started. Three other events were marginal detections of very weak events. Although their identification is probably correct, the determination of their properties (size, duration, ....) is influenced too much by noise for further analysis. Table 1 gives an overview of the final selection of the events retained for further analysis.

In Fig. 2 we show a scatterplot of the spatial extent of the detected brightenings as a function of their peak 
Table 1. Overview of the 41 detected ARTBs. In the second column, we give an appreciation on the detection. Entries in italics correspond to events that are not retained for further analysis. Some events at the beginning and the end of the image sequence are partially missed ("part."). Other events are too much influenced by noise ("noisy") or are purely instrumental ("instr."). In Col. 3, we describe the appearance of the events using the keywords "point like", "single loop" and "multiple loops" introduced by Shimizu (1994). Within the "multiple" class we distinguish between cases in which the contact point between the loops is near their top ("X"), cases in which the contact point is close to the footpoints ("Y"), cases where the loops are in line with each other ("I") and finally the other cases ("O") which are more complicated. In the fourth column ("identification"), we indicate whether the event was classified as a (F)lare, a (M)icroflare or an (I)ndirect event. In case of a flare we also mention to GOES flare class. Arrows indicate the connection to and from indirect events with other ARTBs

\begin{tabular}{|c|c|c|c|c|}
\hline $\mathrm{nr}$ & quality & morphology & identification & Fig. \\
\hline 1 & partial & single loop & - & - \\
\hline 2 & partial & multiple Y & - & - \\
\hline 3 & partial & - & - & - \\
\hline 4 & good & multiple I & M & - \\
\hline 5 & partial & single loop & - & - \\
\hline 6 & noisy & - & - & - \\
\hline 7 & partial & single loop & - & - \\
\hline 8 & good & single loop & M & - \\
\hline 9 & good & single loop & $\mathrm{M} \rightarrow 20$ & - \\
\hline 10 & good & single loop & $\mathrm{M} \rightarrow 16$ & Fig. 12 \\
\hline 11 & good & single loop & $\mathrm{M} \rightarrow 15$ & - \\
\hline 12 & noisy & point like & - & - \\
\hline 13 & good & point like & M & - \\
\hline 14 & good & point like & M & Fig. 11 \\
\hline 15 & good & single loop & $\mathrm{I} \leftarrow 11$ & - \\
\hline 16 & good & single loop & $\mathrm{I} \leftarrow 10$ & Fig. 12 \\
\hline 17 & good & multiple $\mathrm{O}$ & F, B2.8 & - \\
\hline 18 & instr. & - & - & - \\
\hline 19 & instr. & - & - & - \\
\hline 20 & good & single loop & $\mathrm{I} \leftarrow 9$ & - \\
\hline 21 & instr. & - & - & - \\
\hline 22 & good & single loop & M & - \\
\hline 23 & good & multiple I,Y & F, B2.8 & Fig. 8 \\
\hline 24 & good & single loop & $\mathrm{M}, \mathrm{I} ?$ & - \\
\hline 25 & noisy & - & - & - \\
\hline 26 & good & single loop & $\mathrm{F}, \mathrm{B} 2.8$ & - \\
\hline 27 & good & single loop & $\mathrm{F}, \mathrm{B} 2.8 \rightarrow 30$ & - \\
\hline 28 & good & single loop & $\mathrm{M}$ & - \\
\hline 29 & good & single loop & M & - \\
\hline 30 & good & single loop & $\mathrm{I} \leftarrow 27$ & - \\
\hline 31 & good & multiple I & M & - \\
\hline 32 & good & single loop & $\mathrm{M}$ & - \\
\hline 33 & good & single loop & $\mathrm{I} \leftarrow 34$ & Fig. 13 \\
\hline 34 & good & multiple $\mathrm{O}$ & $\mathrm{F}, \mathrm{B} 2.5 \rightarrow 33$ & - \\
\hline 35 & good & single loop & $\mathrm{F}, \mathrm{B} 3.6 \rightarrow 41$ & Fig. 9 \\
\hline 36 & partial & single loop & F, B3.6 & - \\
\hline 37 & good & single loop & M & - \\
\hline 38 & good & point like & M & Fig. 10 \\
\hline 39 & noisy & - & - & - \\
\hline 40 & partial & single loop & & - \\
\hline 41 & partial & single loop & $\mathrm{I} \leftarrow 35$ & - \\
\hline
\end{tabular}

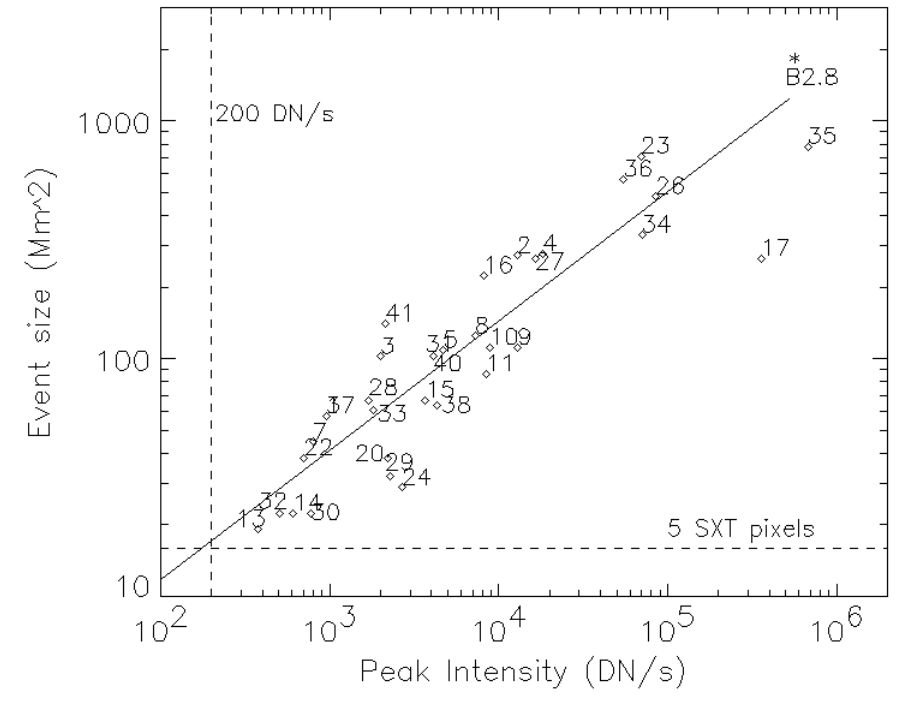

Fig. 2. The size versus the peak intensity of the brightenings detected by SXT in soft X-rays. The plotted peak intensity is the integrated intensity through the $\mathrm{Al} / \mathrm{Mg} / \mathrm{Mn}$ filter over the spatial extent of each event at the time of highest soft X-ray flux, with the background intensity subtracted. The quoted value for the size is the maximal spatial extent of the event during its lifetime. The event numbering refers to Table 1

intensity. The size of the events scales linearly (in $\log / \log$ scale) with a slope of 0.54 . The total range is limited at the high end by the largest/strongest events happening in our dataset (see Sect. 4.1). At the low end, the range is limited by the condition that an event should be detectable in at least 5 pixels at its peak size. Given the peak intensity versus size relation, this 5 pixels condition implies a $200 \mathrm{DN} / \mathrm{s}$ threshold. Let us investigate the limiting factors on the detection of small/weak events. Extrapolating the trend in Fig. 2 suggests that (hypothetical) events of roughly $10 \mathrm{Mm}^{2}$ (say 3 SXT pixels) would have a peak intensity (integrated over the 3 pixels) of the order of $100 \mathrm{DN} / \mathrm{s}$. To be picked up by the detection scheme, we need at least one pixel (the peak position) to qualify at the $5 \sigma_{\mathrm{o}}$ level while the other need to qualify at the $3 \sigma_{\mathrm{o}}$ level. This means that in order to detect this hypothetical event, we need $\sigma_{\mathrm{o}}$ to be lower than $10 \mathrm{DN} / \mathrm{s}$. This is lower than the photon noise alone, in most of the image. The detection of small events is therefore hampered by the signal/noise ratio of the instrument. The minimal size criterium of 5 pixels should be seen as a bypass to avoid the regime in which the limited signal/noise becomes a problem.

In Fig. 3, we show the duration as a function of the size of each of the detected events. The range in durations extends from 1 to nearly $20 \mathrm{~min}$. Although the scatterplot shows a wide spread, a trend from large sizes/large durations to small sizes/short duration is clearly present. The high end of the range in durations is limited by the total duration of our SXT data sequence (36 min). In order to detect a brightening, one needs a reference "quiet" time before and after the brightening to estimate the background intensity from. Indeed, a part of the active region that is bright during the whole observation sequence 


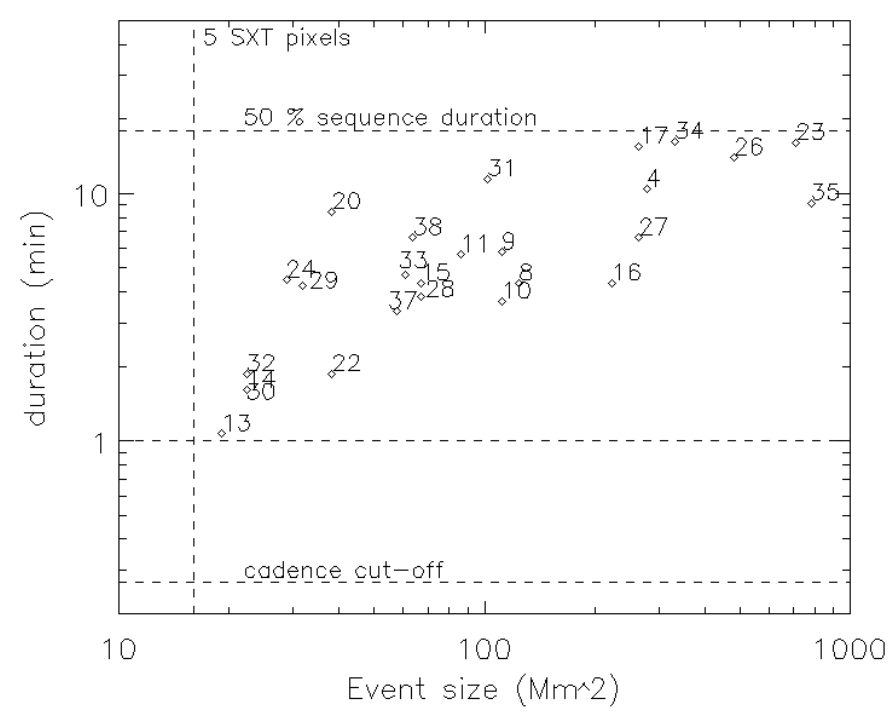

Fig. 3. The duration versus the size of the events detected in the SXT sequence. The quoted value for the size is the maximal spatial extent of the event during its lifetime. The event numbering refers to Table 1. Events whose duration is truncated at the beginning or end of the sequence ("partials" in Table 1) have been omitted from this graph

cannot be identified as a "brightening". Hence the high end cut-off at a fraction of the total duration of the data sequence. Given our image cadence of $8 \mathrm{~s}$, and taking into account that we require a brightening to be present in at least two images, it is in principle possible to detect events as short as $16 \mathrm{~s}$. This is indicated in Fig. 3 as the "cadence cut-off". It is striking to see however, that no events are found with a duration shorter than $1 \mathrm{~min}$. Given the trend of shorter durations for smaller events, this 1 min cut-off may be a consequence of our criterion that an event should extend over at least 5 SXT pixels.

The 1 min cut-off is again a consequence of the instrumental limitations. In Fig. 4, we show the duration of the detected events as a function of their peak temperature.

Using the so-called RTV scaling laws (Rosner et al. (1978), Serio et al. (1991) have estimated the "thermodynamic decay time" of a flaring coronal loop with (full) length $L$ (in units of $\mathrm{Mm}$ ) and a temperature $T$ (in units of MK) as

$\tau=6 \sqrt{10} L T^{-0.5}$,

which is essentially proportional to the conductive cooling time. The dash-dotted curves in Fig. 4 show $\tau$ as a function of temperature for loops with a full length of 3,5 and 10 SXT pixels. Nearly all events are above the curve corresponding to a length of 5 SXT pixels, which is compatible with our detection condition that events should at least have a size of 5 SXT pixels.

The derivation of Eq. (1) requires several assumptions which are not necessarily fulfilled in the present case, such as a single static loop being heated by a single energy release. The events in Fig. 4 may consist of several energy releases in interacting loops. Moreover their measured duration does not only involve the decay phase but also the

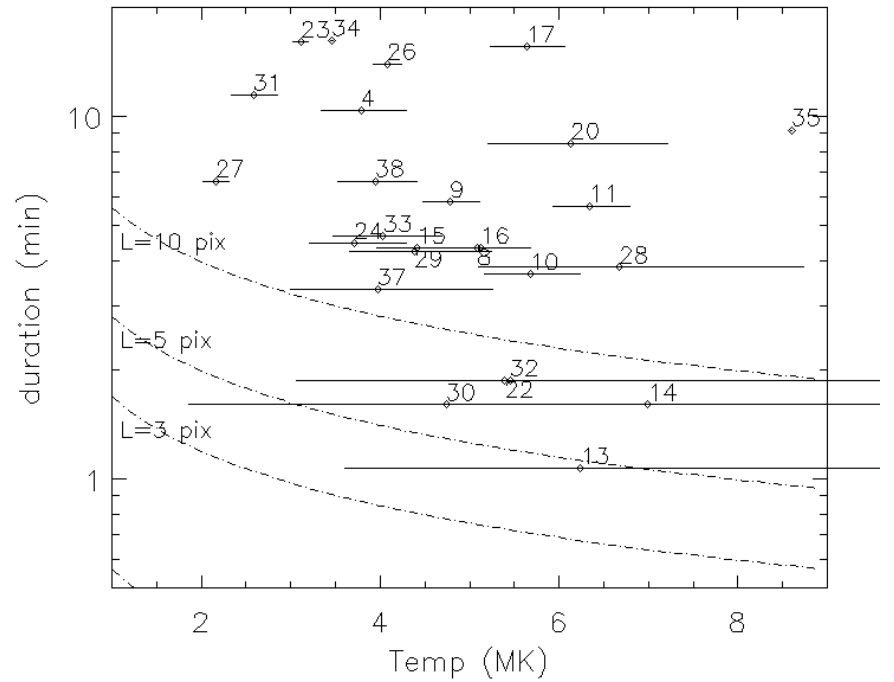

Fig. 4. The duration versus the weighed time-averaged temperature of the events detected in the SXT sequence. The error on the event durations is of the order of $8 \mathrm{~s}$. The event numbering refers to Table 1. Events whose duration is truncated at the beginning or end of the sequence ("partials" in Table 1) have been omitted from this graph. The dot-dashed curves correspond to Eq. (1) for different values of $L$. The "cadence cut-off" (Fig. 3) is outside the vertical range of this graph

rising phase. Yet, these general trend of the curves give physical insight in why detecting events becomes more difficult as one wants to catch events with shorter durations: the main mechanism by which a flaring loop looses its excess energy is by thermal conduction through its footpoints. This means that for short lived events thermal conduction should be operating optimally, which requires loops with short lenghts and high temperatures. For example, for an event to fade in less than $1 \mathrm{~min}$ and having a lenght larger than 5 SXT pixels, a temperature in excess of $7 \mathrm{MK}$ is required to make thermal conduction sufficiently efficient. Therefore, for a fixed minimal spatial dimension (dictated by the instrument spatial resolution, or in our case by the instrumental signal to noise ratio) progressively shorter duration events can be found only in a progressively more restrictive temperature regime. This explains why the few detected events with a duration shorter than 3 min (event 13, 14, 22, 30 and 32), are all very small and relatively hot.

Once the (color) temperature is deduced from the SXT filter pair ratio, we can additionally estimate the thermal energy content (Fig. 5) and a volume emission measure (Fig. 6) for each event. The emission measure (EM) is estimated from the image brightness measured in the thinner filter (Tsuneta et al. 1991), adopting the solar spectrum of Mewe et al. $(1985,1986)$, with the coronal abundances of Meyer (1985). In Fig. 6 we show the volume emission measure versus the temperature of the events.

The thermal energy content is estimated via

$$
\begin{aligned}
E_{\mathrm{th}} & =3 * k_{\mathrm{B}} * T * n_{\mathrm{e}} * V \\
& =3 * k_{\mathrm{B}} * T * \sqrt{E M * A} * \sqrt{d},
\end{aligned}
$$




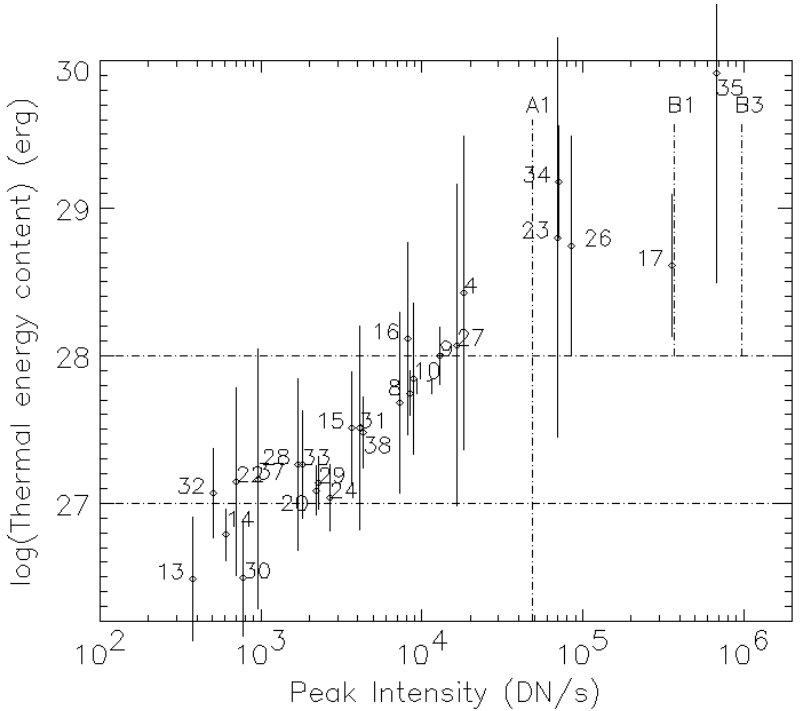

Fig. 5. The peak thermal energy content versus the peak intensity of the brightenings detected by SXT. The plotted peak intensity is the integrated intensity over the spatial extent of each event at the time of highest soft X-ray flux, with the background intensity subtracted. The error bars correspond to a 0.5 sigma uncertainty interval. The event numbering refers to Table 1. Vertical dash-dotted lines refer to the corresponding GOES flare classification. The position of these lines is determined using the relation $\log (S X T)=0.88 \log (G O E S)+11.99$ (Nitta 1997) and taking into account the different SXT filters used

where $k_{\mathrm{B}}$ is Boltzmann's constant, $T$ is the electron temperature, judged from SXT filter ratio, $n_{\mathrm{e}}$ is the electron density, $V$ is the total volume of the emitting plasma, $E M$ is the emission measure calculated from intensity of the SXT signal at the measured temperature, $A$ is the time-varying area of the ARTB in the SXT images, and $d=\sqrt{\max (A(t)) / 3}$ is the line-of-sight depth. For single loop brightenings, it was visually confirmed that this lineof-sight depth formula is a good approximation for the width of the loops. For more complex brightenings (multiple interacting loops), the square root of the area ensures a reasonable scaling. In Fig. 5 we show the thermal energy content as a function of the peak intensity. Most of the events are in the range from $10^{27}$ to $10^{28} \mathrm{erg}$, which is the "microflare" range. The strongest events (top right corner) qualify as A and B flares in the GOES flare qualification (see Sect. 4.1).

The main conclusion that we want to extract from the preceeding scatter-plots is that the transient soft X-ray brightenings observed in active region NOAA 8218 have a range in physical characteristics (duration, size, peak intensity, temperature and emission measure) that is compatible with those reported by Shimizu (1995). Some differences between our set of detected events and the ARTB of Shimizu, such as a higher occurrence rate and perhaps a larger fraction of the weakest events, can probably be attributed to the more sensitive detection scheme that we employed. Just as Shimizu found, the strongest of our

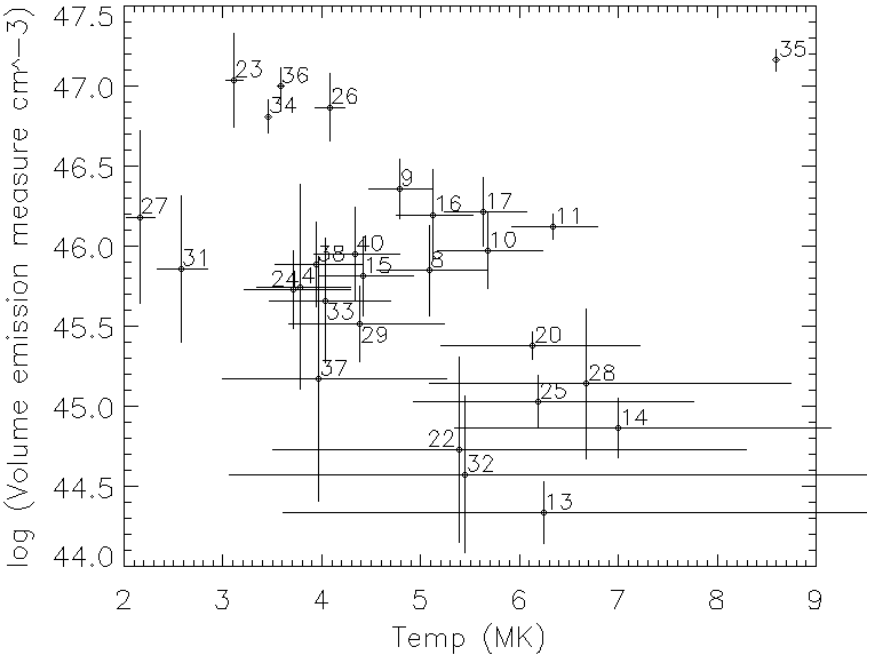

Fig. 6. The volume emission measure integrated over the whole event at the time of the X-ray peak intensity versus weighed time-average-temperature of the brightenings detected by SXT in soft X-rays. The error bars show the 1 sigma uncertainty interval. The event numbering refers to Table 1

events correspond to increases in the X-ray readout of the GOES- 8 satellites. We conclude therefore that the transient soft X-ray brightenings that our scheme detects can indeed be identified as belonging to the same population of transient brightenings as those discussed by Shimizu.

It should be emphasized that the temperatures, and thus emission meausures and energy contents, derived from SXT images depend on some rather important assumptions. First, it is implicitly assumed that each pixel samples a region of isothermal plasma; i.e., a filling factor of unity is assumed. Secondly, since the two images which are used to calculate the filter ratio are not truly simultaneous (in the present case, differing in time by as much as $40 \mathrm{~s}$ ), it must be assumed that the plasma conditions vary slowly enough to make the time mismatch between thin- and thick-filter images unimportant. Clearly, both of these assumptions can be questionned in the analysis of ARTBs. Given this, the temperatures and other physical conditions derived therefrom must not be taken at face value; however, the trends they reveal in a large sample of events may be considered to be more robust than any single measurement with its occasional deviations. In this section we have only used the range and trends of these quantities to conclude that the detected brightening events are ARTBs. Moreover, since the characteristics of ARTBs were determined in a nearly identical way (e.g. Shimizu 1995), any systematical deviations of the methods used, is not expected to alter this conclusion.

For backward comparison, we decribe the appearance of the detected events in Table 1 with the same keywords as Shimizu et al. (1994). Shimizu classifies transient brightenings into three categories: (1) point-like brightenings, (2) brightenings of a single loop, and (3) simultaneous brightenings of multiple loops. 
Shimizu (1997) reported 11\% (18\%) pointlike events, $63 \%(42 \%)$ single loop events and $26 \%(40 \%)$ multiple loop events for active region NOAA 7260 (NOAA 6891). We find roughly $10 \%$ pointlike events, $70 \%$ single loop events and $20 \%$ multiple loop events. Taking into account our limited number of events and the intrinsic difference between various active regions, this is in fair agreement with the fractions reported by Shimizu.

\section{Comparison with EUV counterparts}

In a previous (EUV only) study (Berghmans \& Clette 1999), we have applied the automated detection scheme on the EIT data of SOHO JOP 80. Using the same threshold as the ones used in the previous section on the SXT data (events with a peak intensity exceeding the background level at the $\Sigma_{\mathrm{P}}=5 \sigma_{\mathrm{o}}$ significance level, spatial extent estimated at the $\Sigma_{\mathrm{E}}=3 \sigma_{\mathrm{o}}$ level and duration estimated at the $\Sigma_{\mathrm{D}}=2 \sigma_{\mathrm{o}}$ significance level), we found 373 events. Although these thresholds resulted in well estimated dimensions for most of the events, we noted that the strongest events in the center of the active region tended to merge with smaller neighbours. We therefore repeated the detection procedure using the higher thresholds $\left(\Sigma_{\mathrm{P}}=10 \sigma_{\mathrm{o}}, \Sigma_{\mathrm{E}}=6 \sigma_{\mathrm{o}}, \Sigma_{\mathrm{D}}=4 \sigma_{\mathrm{o}}\right)$ which yield a more appropriate estimate of the dimensions of the 68 strongest events.

In Fig. 1 we have plotted the location of the ARTBs detected in the SXT sequence (squares) as well as the 68 EIT events detected at the high thresholds (crosses), on an MDI magnetogram. The active region consists of a leading negative flux region containing two well-developped sunspots and a more diffuse trailing positive flux region. The two main sunspots are surrounded by patches of the opposite (i.e. positive) polarity. It can cleary be seen that virtually all SXT events (squares) as well as most of the strongest EIT events (crosses) originate at the neutral line between the main sunspots and these nearby islands of opposite polarity.

The weaker EIT events (that are only selected at the low thresholds and not with the higher thresholds) are spread more randomly over the field of view (see Fig. 3 in Berghmans \& Clette 1999). After having carefully checked the location of a number of these weak EIT events in the SXT data, we can confirm that they show in fact no soft X-ray signature at all. Although these weak EIT events are often irregularly shaped, the corresponding TRACE data shows small but clear looplike structures, confirming that these are not false detections. Given the better spatial correspondence with the ARTB in the soft X-rays, we will focus our attention in what follows to the 68 EIT events detected at the high thresholds.

On average, the ARTBs observed with SXT last $5.3 \mathrm{~min}$ and become $145 \mathrm{Mm}^{2}$ large. In comparison, the brightenings detected at the high thresholds seen with EIT last on average longer $(6.1 \mathrm{~min})$ but remain smaller (78 $\mathrm{Mm}^{2}$, on average). Since these average event durations are well above the image cadence (SXT: $8 \mathrm{~s}$, EIT: $15 \mathrm{~s}$ ) and the average event sizes are well above the spatial resolution (pixels are roughly $4 \mathrm{Mm}^{2}$ for both instruments), these differences must reflect a true difference in the properties of the two populations. In fact, we will see in the next section, that in many cases, an ARTB seen in soft $\mathrm{X}$-rays is preceeded by a smaller EUV brightening at the footpoints of the ARTB, which still lives on after their soft $\mathrm{X}$-ray component has faded.

By comparing the EUV and soft X-ray signature of each individual event we identified three different classes of ARTB, which we term "small flares", "microflares", and "indirect events".

\subsection{Small flares}

The soft X-ray flux monitors aboard the GOES satellites of the National Oceanic and Atmospheric Administration (NOAA, US) continuously observe the total soft X-ray flux $(0.5-4 \AA$ and $1-8 \AA)$, integrated over the full solar disc. The $1-8 \AA$ data is broadly accepted as the reference for the X-ray classification of flares, with the letters A, B, C, M and $X$ corresponding to magnitude classes of peak intensity from $10^{-8}(\mathrm{~A})$ to $10^{-4}(X)\left(\mathrm{W} / \mathrm{m}^{2}\right)$.

The upper curve of Fig. 7 shows the recorded GOES-8 readout during the time frame of the SOHO JOP 80 . Two events stand out most clearly:

- a B2.8 flare, (peak: $17^{\mathrm{h}} 37^{\mathrm{m}}$ ),

- a B3.6 flare, (peak: $17^{\mathrm{h}} 56^{\mathrm{m}}$ ).

The lower curves of Fig. 7 show the soft X-ray and EUV intensity as observed by SXT and EIT, integrated over their respective field of view around AR 8218.

Although the GOES detectors have no spatial resolution, the close match of the peaks in the GOES, EIT and SXT data, makes it possible to assess that the GOES peaks did originate from AR 8218. A careful investigation reveals that there is no single detected event that corresponds uniquely to the B2.8 flare. On the contrary, at the time of the B2.8 flare, there are 4 big ARTBs that peak exactly at the same time (to within the $8 \mathrm{~s}$ of the image cadence). ARTB 17 contributes the bulk (68\%) of the soft X-ray peak flux, but also ARTB $23(13 \%)$, ARTB 26 $(16 \%)$ and event $27(2 \%)$ contribute a significant fraction. Note that combining the size and peak intensity of events 17, 23, 26 and 27 in Fig. 2, "corrects" the outlier 17 back to the main trend (indicated by "* B2.8").

In Fig. 8 we show ARTB 23. Note that the loop structure changes during the event. In the third SXT subfield, we see two small bright loops in line with each other and sharing 1 footpoint (multiple I ARTB). In the fifth SXT subfield, however, this has evolved into 1 small bright loop and $1 \mathrm{big}$ fainter loop sharing the other footpoint (multiple Y ARTB). This changing configuration clearly hints at magnetic reconnection. The corresponding EIT and TRACE images show a much smaller brightening that connects the old with the new contact footpoint. ARTB 26 and 27 correspond to the EIT events shown in Fig. 8 of Berghmans \& Clette (1999). 


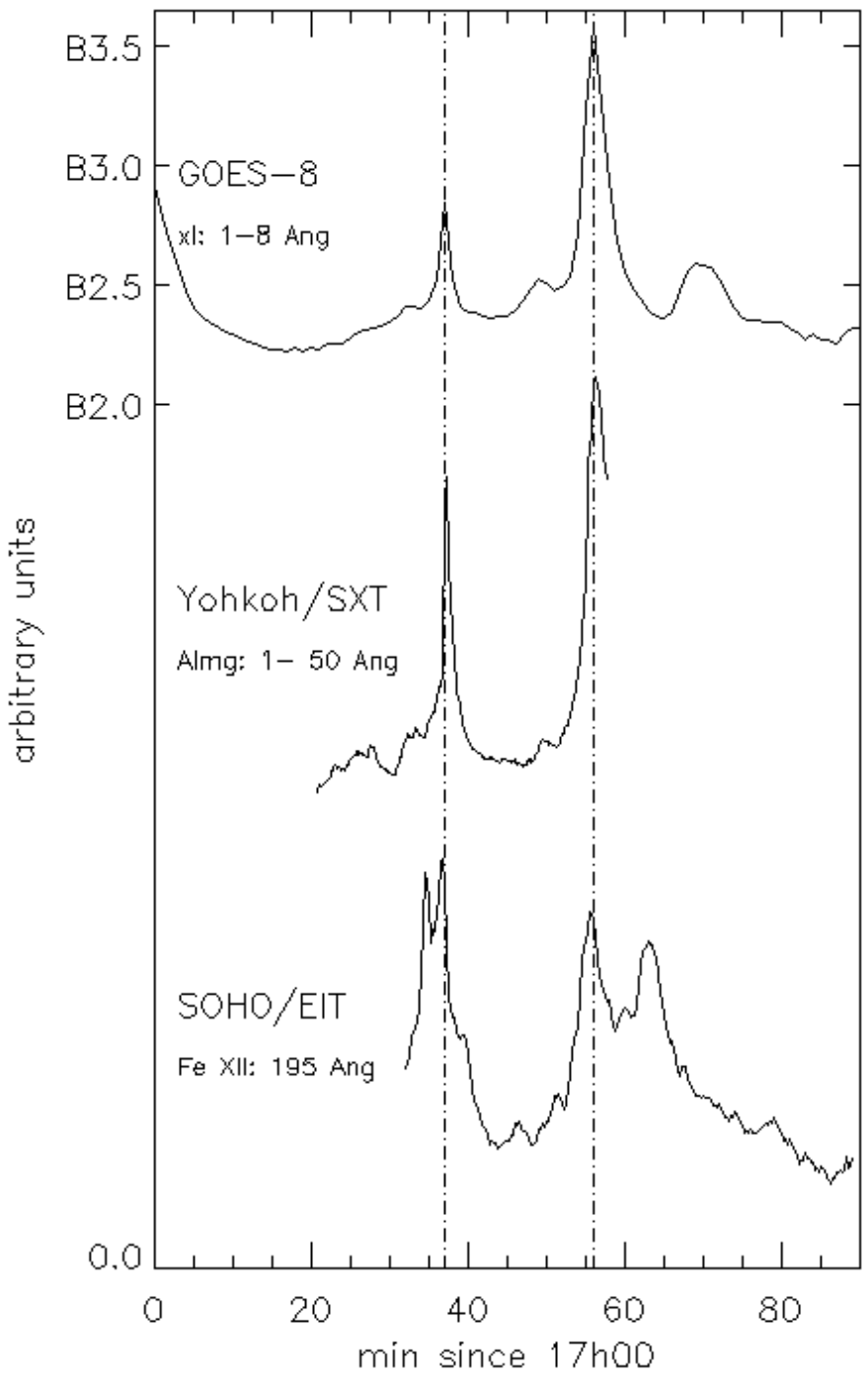

Fig. 7. Spatially integrated intensities from different instruments

For the B3.5 flare, the situation is more simple: only ARTB 35 peaks at the GOES peak flux time. Event 36 contributes $7 \%$ percent of the soft X-ray peak flux but peaks almost a minute earlier. In Fig. 9 we show the early phase in the development of ARTB 35. The event seen in SXT corresponds to a single loop brightening from 1 footpoint, while EIT, and especially TRACE shows, several smaller loops brightening at the footpoints of the SXT loop. The footpoint brightening in EIT started 1 min earlier than the SXT event.

From the ratio of the GOES channels (1-8 $\AA / 0.5-4 \AA)$, estimates can be made for the temperature and the emission measure of the flaring plasma (Garcia 1994). For the B3.5 flare, we find a temperatue of $8.5 \mathrm{MK}$ and a volume emission measure of $10^{47.13} \mathrm{~cm}^{-3}$, in nearly perfect agreement with the estimates obtained from the SXT filter ratio (see event 35 in Fig. 6). For the B2.8 flare, we find from the GOES channel ratio a temperature of $6.38 \mathrm{MK}$ and a volume emission measure of $10^{47.10} \mathrm{~cm}^{-3}$. Since the B2.8 flare is a superposition of several ARTBs, the comparison is less straightforward.

To conclude, the largest ARTBs in our dataset qualify as being $\mathrm{A}$ and $\mathrm{B}$ flares in the GOES classification. These events, which we call "small flares" are found in the top right corner of Fig. 5, above $10^{28}$ erg and with a peak intensity above $10^{4} \mathrm{DN} / \mathrm{s}$. Their sizes exceed $200 \mathrm{Mm}^{2}$. They are all originating near the neutral lines between the main sunspots and the surrounding islands of opposite flux. The flares detected as peaks in the GOES 1-8 A flux, correspond to the combined effect of several ARTBs seen by SXT. Similarly, several EIT brightenings can usually be attributed to each SXT brightening.

\subsection{Microflares}

From Fig. 5 it is clear that below the smallest GOES flare category, the A1 level, the family of detected brightenings extends further down and includes many events with a thermal energy content in the order of $10^{27}$ to $10^{28}$ ergs. By comparing the EUV and the soft X-ray signature of these events, we identified two groups. Some events are "flarelike" in the sense that their time evolution is impulsive and that they have signatures in both EUV and soft X-rays. Given the thermal energy content of the order of $10^{27}$ ergs (a fraction $10^{-6}$ of the largest flare energies around $10^{33} \mathrm{ergs}$ ), these events are identified as "microflares". Other events are more gradual and have a soft $\mathrm{X}$-ray signature only. These will be discussed in the next subsection.

The events that we identify as "microflares" show soft X-ray brightenings that are in the range of $20 \mathrm{Mm}^{2}$ (e.g. Fig. 11) to $100 \mathrm{Mm}^{2}$. These soft X-ray brightenings have the appearance of small loops (Table 1 morphology: "single loop" or "point like") that pre-existed before the brightening starts. Many of these microflares bridge the neutral line just around the main sunspot. The peak soft $\mathrm{X}$-ray intensity recorded by SXT is in the range from several hundred to several thousand $\mathrm{DN} / \mathrm{s}$.

When simulatenous data is available, these events are also detected in the EUV by EIT (195 $\AA$ ). The brightenings observed by EIT have roughly the same appearance as the soft X-ray counterparts but are often somewhat smaller (Fig. 10). TRACE, at $171 \AA$, also observes simultaneous brightenings, but these are much smaller. The TRACE brightenings again have the appearance of small loops located near the center of the SXT brightening (Fig. 10). Although the microflare-events discussed in this subsection are weaker and smaller than the flareevents, they are not especially cooler. From the SXT filter ratios (Fig. 4), we estimate e.g. the temperature of ARTB 14 (Fig. 11) to be above $5 \mathrm{MK}$.

\subsection{Indirect events}

Up till now, we have focused our attention on the flarelike events that are located around the core sunspots of NOAA 8218. From this location, a bundle of loops arches 

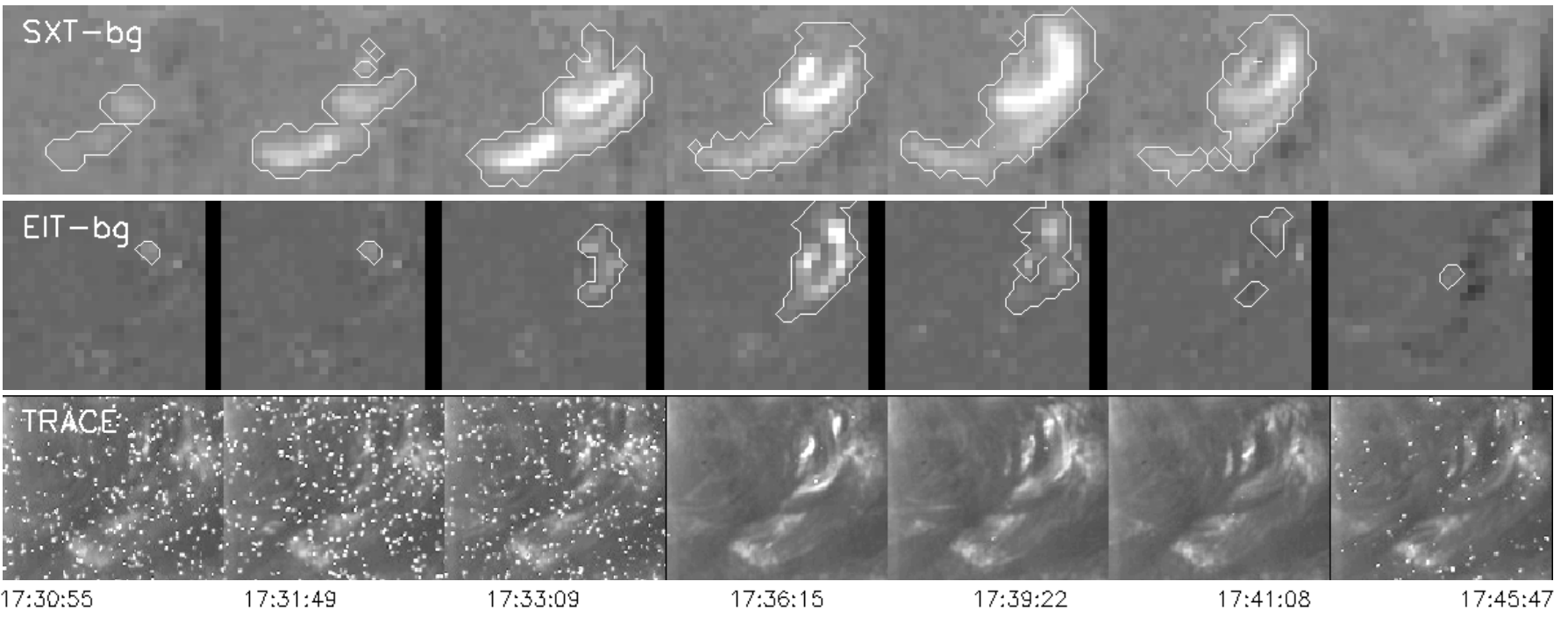

Fig. 8. Time evolution (left to right) of a subfield of 70 arcsec by 60 arcsec around ARTB 23. A slowly varying background (bg) has been subtracted from the SXT subimages (top row) and the EIT subimages (middle row). The white contours in the SXT and EIT subimages encircle events that were detected with the automated algorithm. The TRACE subimages (bottom row) were deliberately not corrected for particle radiation spikes. The time-tags correspond to the TRACE observation times. The EIT and SXT subimages can be off by at most 7 and 4 s, respectively. All the following figures showing time evolutions of subfield will follow this same format
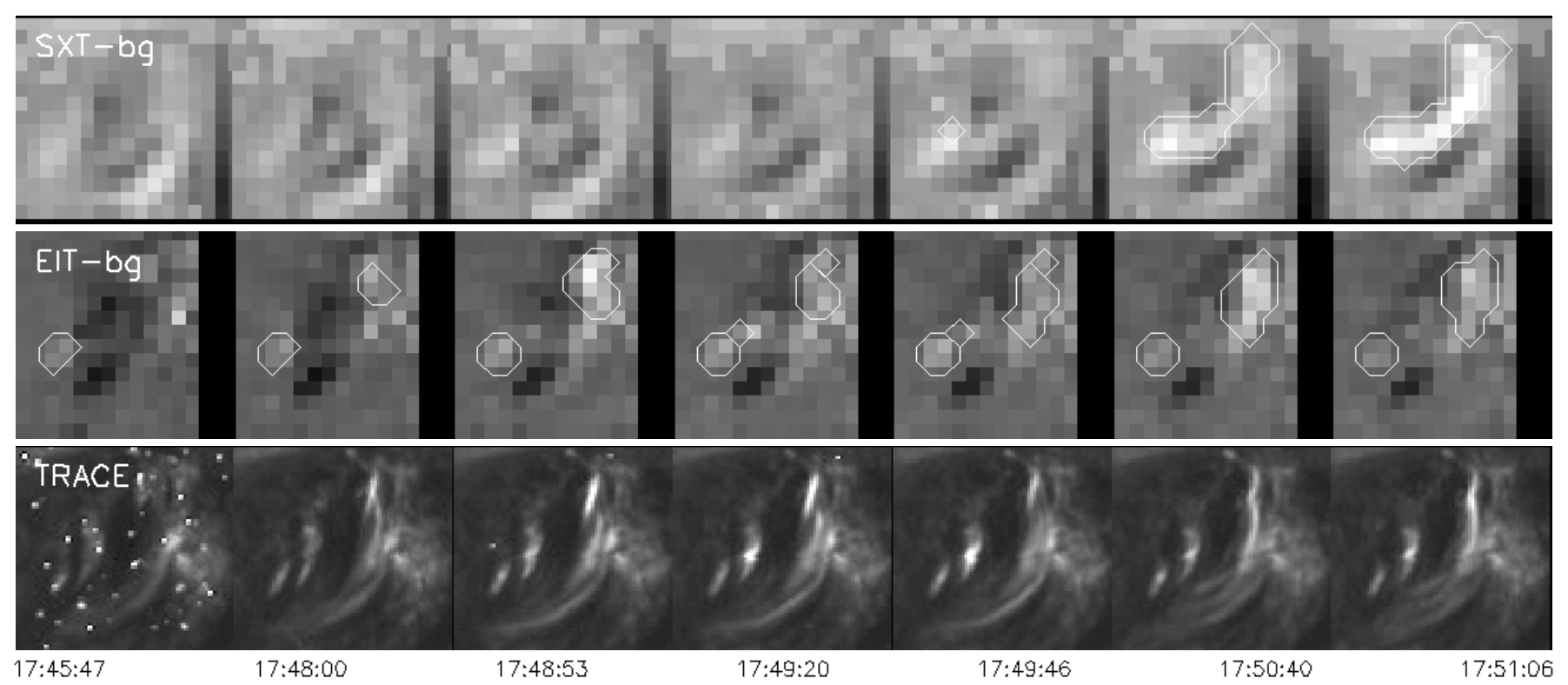

Fig. 9. Time evolution (left to right) of a subfield of 40 arcsec squared around the source of ARTB 35 which is the largest event detected in our dataset (a B3.6 flare). Later on, this ARTB continued peaking in intensity and saturated the detector over a large fraction of the field of view

toward the trailing, positive polarity zone. At the footpoints of these longer SXT loops (see Fig. 1), another type of ARTBs line up: ARTB 16, 33, 20, 15, 30 and 41. These are all located in or near to so-called "moss" regions in the TRACE $171 \AA$ images (Berger et al. 1999), where the hotter loops seen in SXT have their trailing footpoints. In contrast to the more impulsive events in the previous sections, these events consist of the moderate brightening of some larger X-ray loops, followed by a slow fading. The enhancement is more pronounced towards the loop's footpoints but can be found over a large part of the loop (e.g. Fig. 13).
A striking characteristic is that no signature at all is found in the EUV. This suggests that these events are not localised reconnection sites bringing plasma to coronal temperatures, but rather a gradual evolution of preexisting SXT loops.

We speculate that these moderate brightenings are a by-product of the microflaring activity at the other footpoint near the core sunspots. A clear example is shown in Fig. 12. The event on the right of the subimage at $17 \mathrm{~h} 24 \mathrm{~m} 57 \mathrm{~s}$ and $17 \mathrm{~h} 25 \mathrm{~m} 51 \mathrm{~s}$ is ARTB 10 , which is a microflare both visible in the SXT image as well as in the TRACE image. While ARTB 10 decays, a new event 

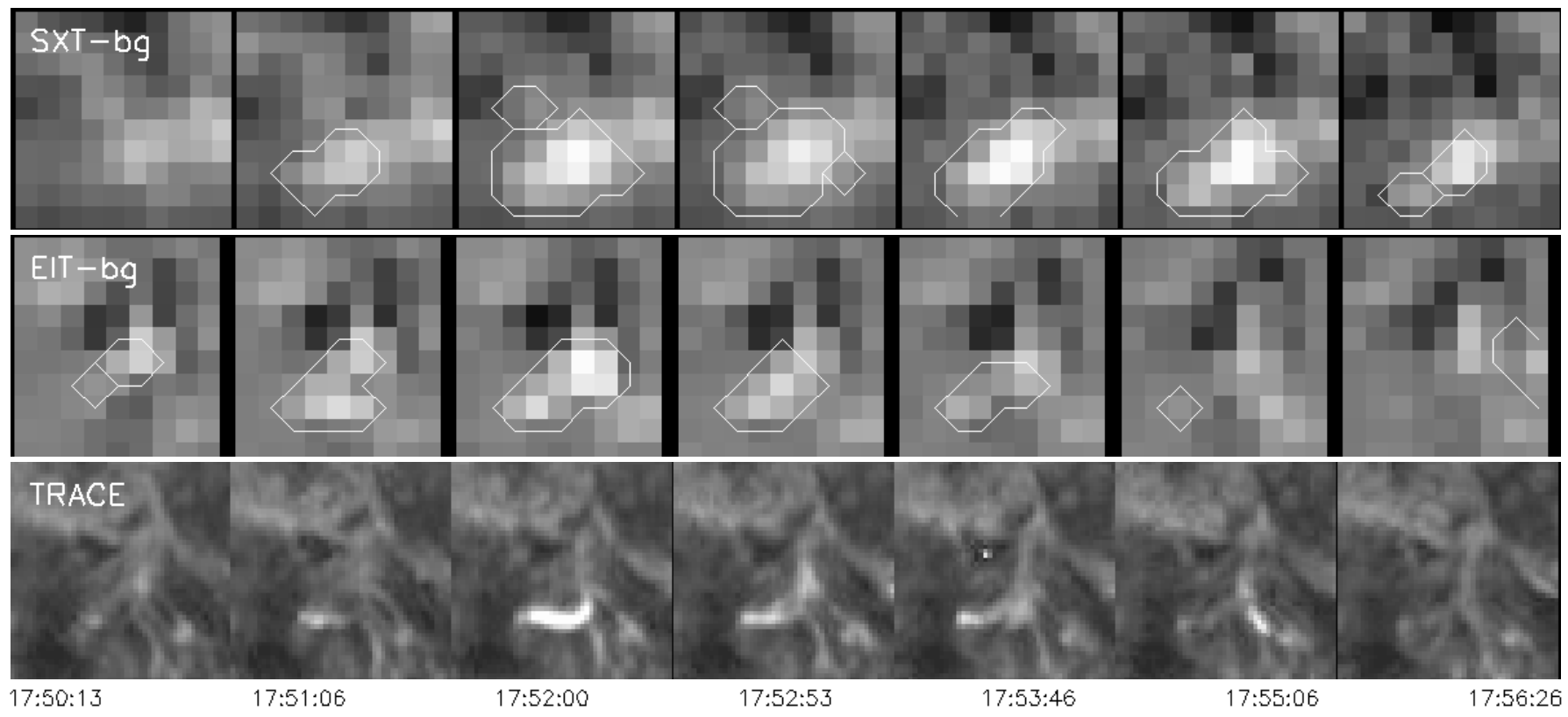

Fig. 10. Time evolution (left to right) of a subfield of 25 arcsec squared around ARTB 38. This event is shown as a prototype in its category of microflares: a small somewhat elongated brightening, with a similar appearance in the SXT and the EIT image, whereas at the TRACE resolution one or two loops can be seen. The event is located (Fig. 1) just on the neutral around one of the main sunspots of the active region
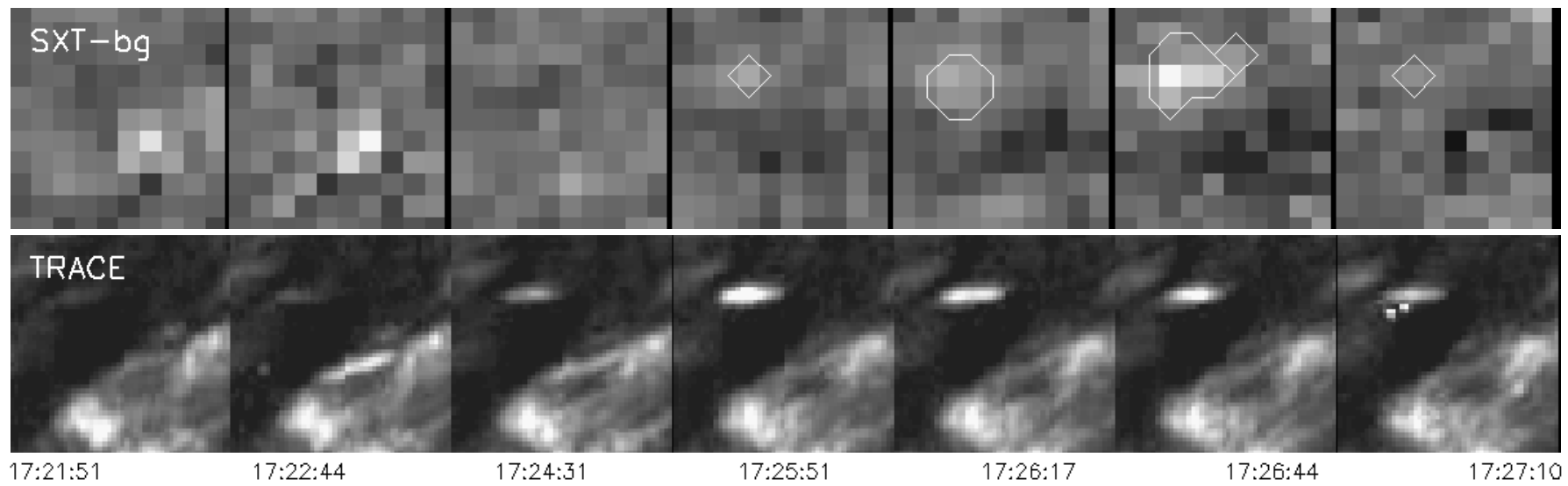

Fig. 11. Time evolution (left to right) of a subfield of 25 arcsec squared around ARTB 14 which is among the smallest brightenings detected. The corresponding loop that brightens in the simultaneous TRACE images shows that this brightening was a true solar event and not a noise fluctuation. Note that the first two SXT subimages contain a brightening of $2-3$ pixels which was not detected. Again the corresponding TRACE loop suggests a true solar event

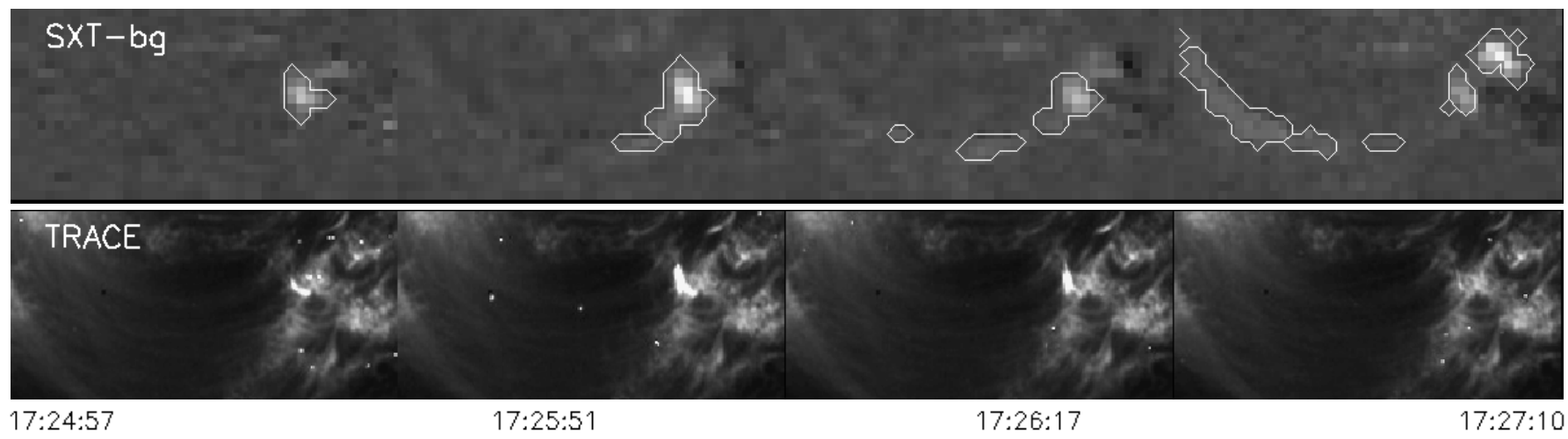

Fig. 12. Time evolution (left to right) of a subfield of 110 arcsec by $55 \operatorname{arcsec}$ showing at the right ARTB 10 and on the left ARTB 16. The perturbation travels from the right footpoint to the left footpoint at a speed of $310 \mathrm{~km} \mathrm{~s}^{-1}$. A time profile along the propagation path is shown in Fig. 14 

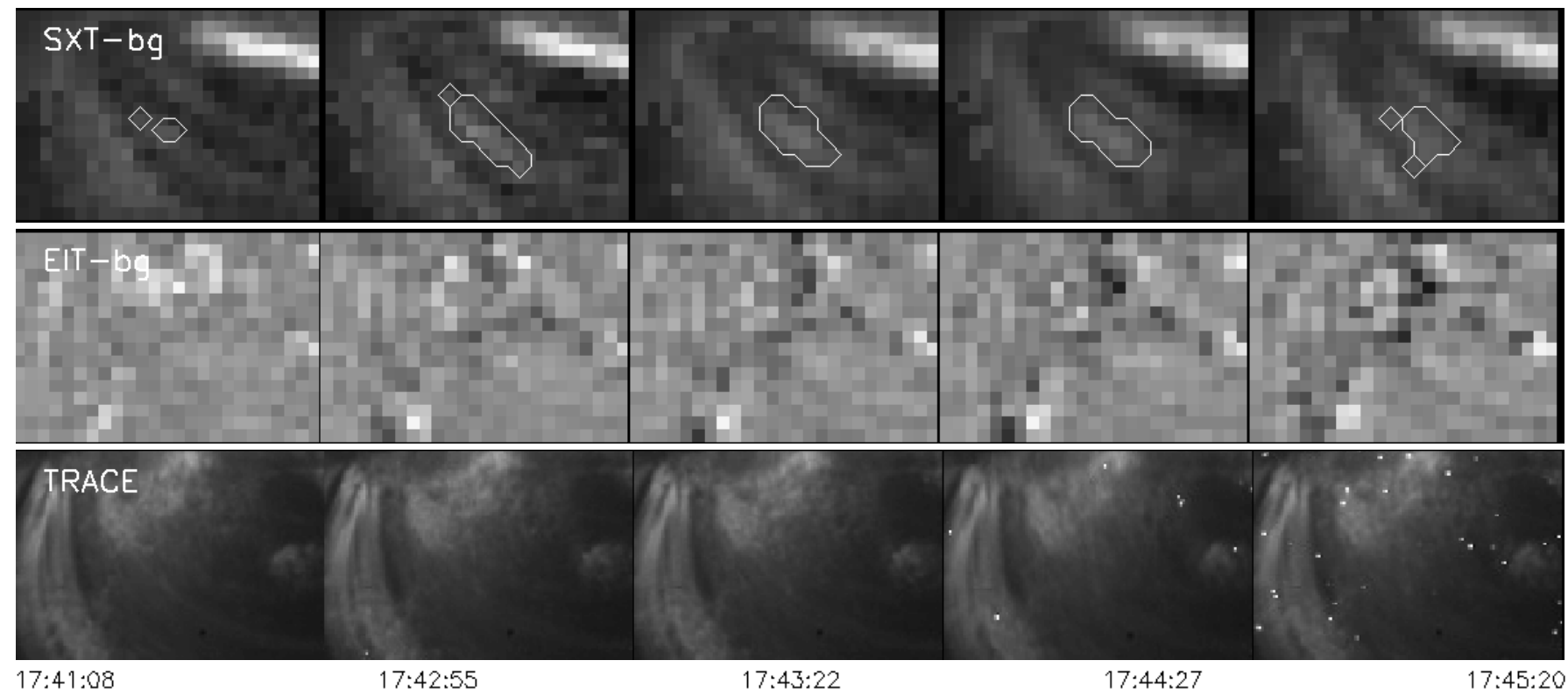

Fig. 13. Time evolution (left to right) of a subfield of 65 arcsec by 45 arcsec around ARTB 33: A non-impulsive brightening which is in many respects similar to ARTB 16 (Fig. 12): a moderate brightening towards a footpoint of a large pre-existing SXT loop. ARTB 34 is the most likely source perturber for this indirect event, though unambiguous identification is not possible in this case

emerges on the lefthand side of the subimage. This is ARTB 16, a gradual brightening of a long pre-existing SXT loop without any signature in the EUV. The relation between ARTB 10 and ARTB 16 becomes especially clear when we follow the time evolution of a path that outlines the loop connecting ARTB 10 and ARTB 16 (Fig. 14). The bright ridge in the figure clearly demonstrates that a bright patch of plasma is ejected from ARTB 10 and travels along the loop. When it reaches the other footpoint, it is identified by our brightening detection scheme as ARTB 16. The nature of the propagating disturbance could be either a flow (like a small X-ray jet) or either an MHD slow mode wave.

From the SXT filter pair, it was estimated that the local temperature during ARTB 16 evolved from $4 \mathrm{MK}$ to a peak value of 5.1 MK. Since the sound speed can be written as a function of temperature only (Priest 1984)

$c_{\mathrm{S}}=152 T^{1 / 2} \mathrm{~m} / \mathrm{s},(T$ in Kelvin $)$

we can translate these temperatures to a range of sound speed from roughly 300 to $350 \mathrm{~km} \mathrm{~s}^{-1}$. The speed of propagation, as measured by tracking the brightening in successive SXT images, is approximately $310 \mathrm{~km} \mathrm{~s}^{-1}$. This seems to suggest that we see are seeing a propagating MHD slow mode wave. If so, the vague indication of a repetition of the bright ridge pattern in Fig. 14, could be explained as wave reflections on the loop's footpoints.

Besides this very clear example of ARTB 10-16, we could indentify 5 more, similar pairs of microflare-indirect events. These pairs are listed in Table 1 (fourth column).

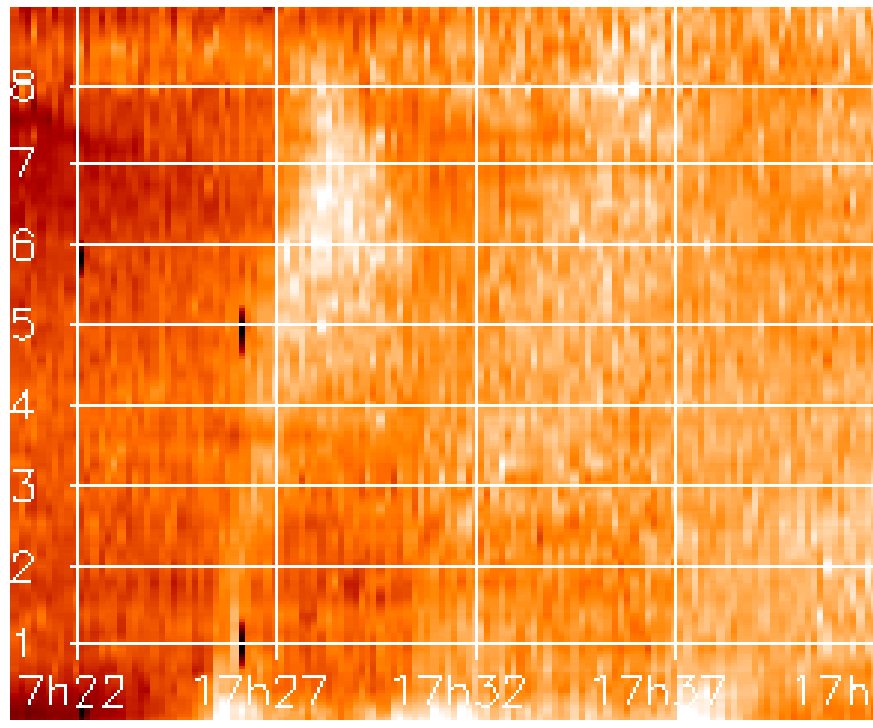

Fig. 14. xt-diagram showing the time evolution (horinzontal axis) of a path (vertical axis) following the loop that connects ARTB 10 and ARTB 16 in the top panel of Fig. 1. The calculated background has been subtracted to enhance the contrast

\section{Discussion}

In this paper we have examined the connection between two types of brightenings frequently observed in active regions. On one side, we have the active region transient brightenings (ARTBs), which are soft X-ray brightenings first detected with SXT (Yohkoh) almost a decade ago (Shimizu 1992). On the other side, we have EUV brightenings that are observed with EIT high-cadence images and with the TRACE imager. Based on an automated detection algorithm applied to simultaneous image 
sequences from these instruments, we present the following results:

- Our detection scheme recovers ARTBs in high cadence SXT data with similar characteristics/dimensions as those described by Shimizu et al. (1992) and Shimizu \& Tsuneta (1997);

- In a simulateneous \& cospatial high cadence EIT sequence, we discover the EUV counterparts of the ARTBs. These are much more numerous by about a factor 5. Most of the weaker EIT events have no soft $\mathrm{X}$-ray counterpart, and were not further considered in this paper (see Berghmans \& Clette 1999; Robbrecht et al. 2001);

- The strongest events seen in the EIT sequence (peak intensity exceeding $10 \sigma_{\mathrm{o}}$ above the background) have a much better spatial correspondence to the ARTBs detected in the SXT sequence. On average, the ARTBs observed with SXT become $145 \mathrm{Mm}^{2}$ large, while the high peak EIT events become only $78 \mathrm{Mm}^{2}$ large. Since the SXT and EIT pixel size (corresponding to less than $4 \mathrm{Mm}^{2}$ on the Sun) are much smaller than these event size, this difference is significant. Analogously the difference in duration of the ARTBs observed with SXT (5.3 $\mathrm{min})$, and the high peak EIT events $(6.1 \mathrm{~min})$, is much larger than the EIT (15 s) and SXT (8 s) image cadence and therefore significant;

- Using simultaneous data from GOES satellites, the strongest ARTBs belong to A and B class flares. There is however no one to one correspondence, as the flares identified by GOES seem to consist of several ARTBs which are spatially separated. The seems to lead to the concept of sympathetic ARTBs, that collectively form a flare as identified by GOES. Analogously, most of these stronger ARTB detected by SXT often corresponds to several EUV brightenings seen by EIT/TRACE;

- Among the weaker ARTBs (below $10^{28}$ ergs), two subpopulations can be identified: microflares and indirect events. The microflares have similar soft X-ray and EUV signatures and have an impulsive time-evolution. They typically originate at the neutral lines between the main sunspot and nearby islands of opposite polarity;

- The indirect events are echos of the flarelike events that propagate along pre-existing SXT loops; they typically occur near "moss" regions (Berger et al. 1999) but have otherwise no EUV signature at all. A useful example of this process is provided by the joint events ARTB 10 and 16 (Figs. 12 and 14), wherein a brightening is apparently ejected from one footpoint of a pre-existing loop and transported to the far footpoint. The propagation speed in this event is comparable to the sound speed which suggests an interpretation in terms of slow mode MHD waves.

In an answer to the questions posed in the introduction, these results show that there is no simple one-to-one correspondence between the ARTB seen with SXT and the
EUV brightenings seen with TRACE and EIT. ARTBs, especially the stronger ones, often correspond to several EUV brightenings. For the weaker "indirect events", there is usually no EUV counterpart at all. In contrast to most of the ARTBs, these indirect events do not seem to belong to the larger "flare family". At the same time, many of the weaker EUV brightenings have no soft X-ray counterpart. We assume that these are events with weaker energy releases for which no plasma is heated beyond $210^{6} \mathrm{~K}$. These transient are thus undetectable in soft X-rays. We found that for the weakest ARTBs, the detection is hampered by the limited signal to noise ratio of the instrument. Our present detection tool scans the image sequence of one instrument at a time. In future work this procedure might be upgraded such that cospatial/cotemporal data from different instruments may be scanned at the same time. This will allow to pick up e.g. very weak soft X-ray brightenings with a sufficiently significant EUV peak such as the minor undetected event shown in Fig. 11.

The limited number of events in the present dataset does not allow for a quantitative determination of the powerlaw distribution of the events as function of their energy. On a qualitative level, this paper has shown that the accurate determination of this powerlaw faces at least two fundamental problems:

- Not every brightening corresponds to a genuine heating event. We have demonstrate that although the indirect, wave-like motions do qualify as ARTBs, they are not flare-like but merely a byproduct of activity ocurring elsewhere. In order to derive accurately the total heating budget, a careful extraction has to be made of the flare-like events among all the detected brightenings. Aschwanden et al. (2000) find that applying such a flare-criterion leads to significant flatter powerlaw distribution (about -1.8) than previous studies (e.g. Krucker \& Benz 1998) that sample all EUV brightenings, regardless whether they are flare-like or not;

- A small flare recorded by GOES as a single peak, can correspond by SXT as several, spatially well separated ARTBs. Each of these ARTB in turn, can correspond to several EUV brightenings. This hierarchy posses a serious problem when trying to count individual events for an energy histrogram. What is an individual event? Neglecting possible associations between nearby brightenings, leads to an overestimation of the number of small events and thus to unrealistically steep powerlaws.

Acknowledgements. SOHO is a project of international cooperation between ESA and NASA. The Yohkoh soft X-ray telescope is a collaborative project of the Lockheed Palo Alto Research Laboratory, the National Astronomical Observatory of Japan, and the University of Tokyo, supported by NASA and ISAS. The kind help from Garcia Howard and Dan Wilkinson (SEC/NOAA) on the GOES data was most apppreciated. TRACE is a mission of the Stanford-Lockheed Institute for Space Research, and part of the NASA Small Explorer 
program. It is a pleasure to acknowledge discussions with Jean-Francois Hochedez. This work was supported by the Belgian Federal Services of Scientific, Technical and Cultural Affairs (SSTC/DWTC). DMcK was supported by NASA under Marshall Space Flight Center contract NAS8-40801 with the Lockheed Martin Advanced Technology Center.

\section{References}

Aschwanden, M. J. 1999, Sol. Phys., 190, 233

Aschwanden, M. J., Tarbell, T. D., Nightingale, R. W., et al. 2000, ApJ, 535, 1047

Berger, T. E., De Pontieu, B., Schrijver, C. J., \& Title, A. M. 1999, ApJL, 519, L97

Berghmans, D., \& Clette, F. 1999, Sol. Phys., 186, 207

Berghmans, D., Clette, F., \& Moses, D. 1998, A\&A, 336, 1039

Bentley 2000, http://www.lmsal.com/ $\sim$ bentley/guides/tag/

Clette, et al. 1998, http://sohowww.nascom.nasa.gov/soc/JOPs/jop080.txt

Crosby, N. B., Aschwanden, M. J., \& Dennis, B. R. 1993, Sol. Phys., 143, 275

Delaboudinière, J.-P., et al. 1995, Solar Phys., 162, 291

Habbal, S. R., Ronan, R., \& Withbroe, G. L. 1985, Solar Phys., 98, 223

Garcia, H. A. 1994, Sol. Phys., 154, 275

Gary, D. E., Hartl, M. D., \& Shimizu, T. 1997, ApJ, 958

Gopalswamy, N., Payne, T. E. W., Schmahl, E. J., et al. 1994, ApJ, 437, 522

Gopalswamy, N., Zhang, J., Kundu, M. R., Schmahl, E. J., \& Lemen, J. R. 1997, ApJ, 491, L115

Handy, B. N., et al., Sol. Phys., 187, 229

Hudson, H. S. 1991, Solar Phys., 133, 357

Kjeldseth-Moe, O., \& Brekke, P. 1998, Solar Phys., 182, 73

Krucker, S., \& Benz, A. O. 1998, ApJ, 501, L213

Krucker, S., Benz, A. O., Bastian, T. S., \& Acton, L. W. 1997, ApJ, 488, 499

Lin, R. P., Schwartz, R. A., Kane, S. R., Pelling, R. M., \& Hurley, K. C. 1984, ApJ, 283, 421

Mason, H. E., Young, P. R., Pike, C. D., et al. 1997, Sol. Phys., 170, 143

Mewe, R., Gronenschild, E. H. B. M., \& van den Oord, G. H. J. 1985, A\&AS, 62, 197
Mewe, R., Lemen, J. R., \& van den Oord, G. H. J. 1986, A\&AS, 63,511

Meyer, J. P. 1985, ApJS, 57, 173

Neupert, W. M. 1968, ApJ, 153, L59

Nindos, A., Kundu, M. R., \& White, S. M. 1999, ApJ, 513, 983

Nitta, N. 1997, ApJ, 491, 402

Nigthingale, R. W., Aschwanden, M. J., \& Hurlburt, N. E. 2000, Sol. Phys., 190, 249

Ogawara, Y., Takano, T., Kato, T., et al. 1991, Sol. Phys., 136,1

Porter, J. G., Toomre, J., \& Gebbie, K. B. 1984, ApJ, 283, 879

Porter, J. G., Fontenla, J. M., \& Simnett, G. M. 1995, ApJ, 438,472

Priest, E. 1984, Solar Magnetohydrodynamics (D. Reidel Publ. Co., Dordrecht, Holland)

Robbrecht, E., Verwichte, E., Berghmans, D., et al. 2001, A\&A, accepted

Rosner, R., Tucker, W. H., \& Vaiana, G. S. 1978, ApJ, 220, 643

Schadee, A., de Jager, C., \& Svestka, Z. 1983, Sol. Phys., 89, 287

Serio, S., Reale, F., Jakimiec, J., Sylwester, B., \& Sylwester, J. 1991, A\&A, 241, 197

Shimizu, T., Tsuneta, S., Acton, L. W., Lemen, J. R., \& Uchida, Y. 1992, PASJ, 44, L147

Shimizu, T., Tsuneta, S., Acton, L. W., Lemen, J. R., Ogawara, Y. \& Uchida, Y. 1994, ApJ, 422, 906

Shimizu, T. 1995, PASJ, 47, 251

Shimizu, T. 1997, Studies of Transient Brightenings (Microflares) Discovered in Solar Active Regions, Ph.D. Thesis, National Astronomical Observatory and University of Tokyo, Japan, chapter 3

Shimizu, T., \& Tsuneta, S. 1997, ApJ, 486, 1045

Tarbell, T. D., et al. 1994, in Proc. 3rd SOHO workshop: Solar Dynamic Phenomena and Solar Wind Consequences (Paris: ESA), ESA SP-373, 375

Tsuneta, S., Acton, L., Bruner, M., et al. 1991, Sol. Phys., 136, 37

Tsuneta, S., \& Lemen, J. R. 1993, in Advances in Stellar and Solar Coronal Physics, ed. J. F. Linsky, \& S. Serio (Dordrecht: Kluwer), 113

White, S. M., Kundu, M. R., Shimizu, T., Shibasaki, K., \& Enome, S. 1995, ApJ, 440, 435

Withbroe, G. L., Habbal, S. R., \& Ronan, R. 1985, Solar Phys., 95, 297 